\section{Suckling Reflex in the Cat}

For experimental purposes, some new-born kittens had to be reared in the laboratory away from the mother. Difficulties were encountered in inducing the kittens to accept milk administered by means of a glass pipette, until a way of evoking the suckling response was found. It was observed that slight pressure from the pipette applied to the premaxillary part of the palate invariably produced the suckling response which was maintained so long as the kitten was disposed to accept food. The main feature of this response is a slight protrusion of the tongue with side-to-side concavity of the dorsum, which assumes the shape of a shoehorn. The nozzle of the pipette is firmly grasped between the tongue and the premaxillary palate. The upturned margins of the more posterior part of the tongue press against the palate. A closed channel is thus formed within the oral cavity. The protruded tip of the tongue works up and down the side of the pipette in a milking action. Suction is created by alternate depression and elevation of the floor of the oral cavity along with the mandible, while the margins of the tongue and the palate together maintain the closed channel.

It will be noted that the mechanism described above differs from that in the human baby, in that the lips take no part at all in sealing the oral cavity off from the exterior. An oral sphincter of the human type does not exist in carnivores.

\section{Department of Anatomy, Faculty of Medicine, \\ University of Malaya, Singapore. \\ July 7.}

\section{A Human Blood-Group Gene Intermediate between $M$ and $N$}

WHILE many blood-group antigens have, subsequently to their discovery, proved to be divisible in. to a number of sub-types, the $M$ and $N$ antigens have shown little tendency to be subdivided. Aberrant $M$ and $N$ antigens have from timo to time been described and attributed to corresponding genes, sometimes characterized as $M_{2}$ and $N_{2}$, but both of these are extremely rare, with frequencies of the order of 1 in 1,000 in all populations hitherto tested. It was therefore with great surprise that we found examples of an abnormal $M$ antigen and also of an abnormal $N$ in a single English family. The results of our tests on members of this family are shown in Table 1. The sera used have each been tested with consistent results against very many-in some cases thousands -other red cell samples. The classification of the sera into positive and negative reactors with members of the C. family depends solely on tests on mem. bers of that family and on incomplete tests on one other unrelated person. All the tests have been carried out at least in duplicate, and in the case of Mrs. C. and K. C. tests have been done on separate blood samples at intervals of several months.

It will be seen that the red cells of Mrs. C. and P. C. react with all anti- $M$ sera, but with only three out of twenty-four rabbit anti- $N$ and two out of four human anti- $N$ sera, while those of $\mathrm{K}$. C. react with all anti- $N$ sera and with all but one of twenty-one rabbit anti- $M$, and all but one of six human anti- $M$
Table 1. REACtions of RED Chlls of C. Family with ANTI- $M$ Table 1. REACTIONS OF RED CHLLS OF C. FAMI

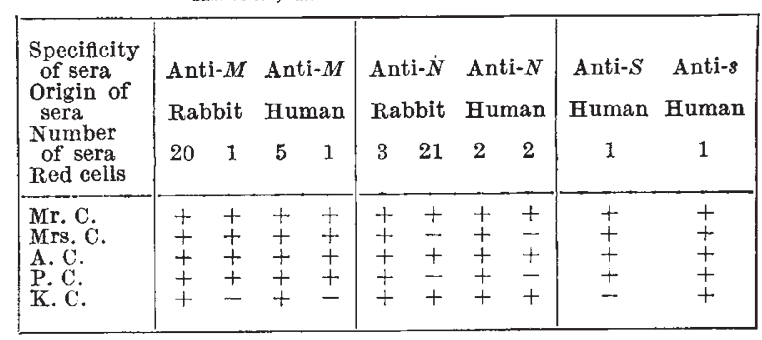

sera. The Henshaw and Hunter antigens ${ }^{1}$ which form part of the MNSs complex were shown to be absent from the red cells of all five persons. The wife and daughter of K. C. were both shown to be of the normal genotype $N s N s$.

Accepting the stated relationships of the persons tested, the hypothesis of separate $M_{2}$ and $N_{2}$ genes occurring in the one family is genetically impossible, since K.C. must have obtained his normal $N$ from his father and his abnormal $M$ from his mother, who, however, has a normal $M$ and an abnormal $N$. The occurrence of the very rare $N_{2}$ and of a mutation from $M$ to $M_{2}$ in a single family is too highly improbable to be considered.

Samples of the anti- $M$ sera which agglutinated the red cells of K. C. were absorbed with normal $M$ and $M N$ cells and were then found not to agglutinate the K. C. cells. This shows that the agglutination of the cells of $\mathrm{K}$. C. cannot be due to an extra antibody in the anti- $M$ sera, corresponding to some rare antigen independent of the $M N$ system. It was similarly shown that the agglutination of the cells of Mrs. C. and P.C. was not due to an additional antibody in the anti- $N$ sera.

No genetically tenable scheme of modifying genes appears capable of accounting for the phenomena.

The only hypothesis which we have been able to formulate to account for our observations depends on the occurrence of a gene having properties intermediate between those of the $M$ and the $N$ genes, and allelic to these. If we provisionally call this gene $M^{c}$, then it is assumed that red cells of genotype $M M^{c}$ will be agglutinated by all anti- $M$ sera and by some, but not all, anti- $N$ sera, whereas those of genotype $N M^{c}$ will be agglutinated by all anti- $N$ sera but only by some anti- $M$ sera. The gene $M^{c}$, being an allele of $M$ and $N$, will be closely linked to $S$ or $s$. Sera were fortunately available to test all members of the family with anti- $S$ and anti-s, and the reactions of the cells of the five persons with each of these two sera provide a critical test of the hypothesis. The reactions of all members of the family can be accounted for in full by assigning them the genotypes shown in the accompanying diagram.

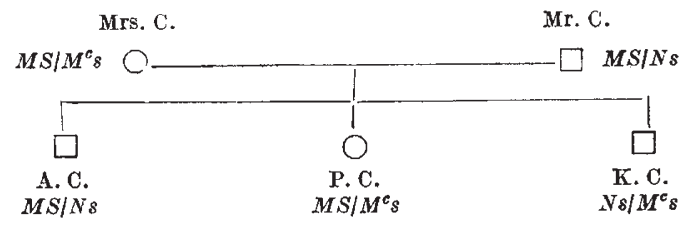

The $M N S \&$ genotypes of the C. family

The properties here attributed to the $M^{c}$ gene are almost completely analogous with those of the $c^{v}$ gene in the $R h$ system $^{2}$. 